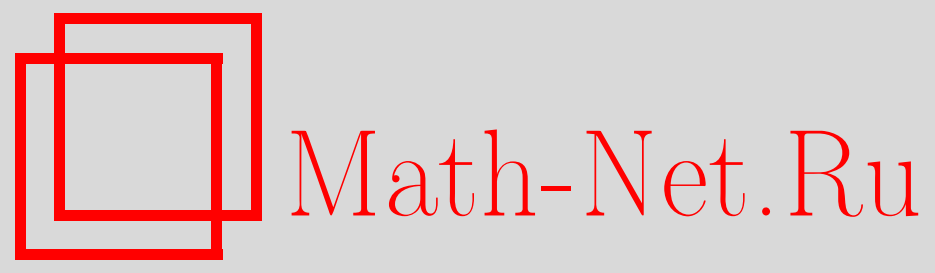

Ю. Л. Павлов, Предельные теоремы для объемов деревьев в случайном непомеченном лесе, Дискрет. матем., 2005, том 17, выпуск 2, 70-86

DOI: https://doi.org/10.4213/dm99

Использование Общероссийского математического портала Math-Net.Ru подразумевает, что вы прочитали и согласны с пользовательским соглашением http://www . mathnet.ru/rus/agreement

Параметры загрузки:

IP: 35.173 .137 .237

26 апреля 2023 г., 12:59:38 


\title{
Предельные теоремы для объемов деревьев в случайном непомеченном лесе
}

๑) 2005 г. . Л. Павлов

\begin{abstract}
Для случайного леса, состоящего из $N$ деревьев и $n$ непомеченных вершин, получено описание предельного поведения максимального объема дерева и числа деревьев заданного объема во всех зонах стремления $N$ и $n$ к бесконечности.

Работа выполнена при поддержке программой Президента Российской Федерации поддержки ведущих научных школ Российской Федерации, грант НШ 1758.2003.1.
\end{abstract}

В работах $[1,2,3]$ рассматривались леса, состоящие из $N$ корневых деревьев и содержащие $n$ некорневых вершин, при этом корни деревьев занумерованы числами от 1 до $N$, а некорневые вершины - числами от 1 до $n$. На множестве всех таких лесов вводилось равномерное распределение вероятностей, и при $N, n \rightarrow \infty$ было получено описание предельного поведения максимального объема дерева и числа деревьев заданного объема. Ниже будут доказаны аналогичные результаты для случайных корневых лесов с незанумерованными вершинами. Обозначим через $F_{N, n}$ множество лесов, состоящих из $N$ корневых деревьев, упорядоченных одним из $N$ ! возможных способов, и $n$ некорневых непомеченных вершин. Пусть на $F_{N, n}$ задано равномерное распределение вероятностей. Под объемом дерева в лесе из $F_{N, n}$ будем понимать число некорневых вершин, содержащихся в этом дереве.

Задача перечисления непомеченных деревьев изучалась в работе [4]. Введем производящую функщию

$$
T(x)=\sum_{k=1}^{\infty} T_{k} x^{k}
$$

где $T_{k}$ - число корневых непомеченных деревьев, содержащих $k$ вершин, включая корень. В [4] рассматривались свойства этой функции и показано, что

$$
T(x)=x+x^{2}+2 x^{3}+4 x^{4}+9 x^{5}+\ldots
$$

и эта производящая функция имеет радиус сходимости $R=0,3383219 \ldots$ Кроме того, согласно [4],

$$
T(R)=1
$$

и функщия $T(x)$ может быть представлена в виде

$$
T(x)=1-b(R-x)^{1 / 2}+b_{1}(R-x)+b_{2}(R-x)^{3 / 2}+\ldots,
$$


числовые коэффищиенты $b, b_{1}, b_{2}, \ldots$ определены в [4], в частности, $b=2,681127 \ldots$ В [4] также установлено, что при $k \rightarrow \infty$

$$
T_{k}=\beta\left(R^{k} k^{3 / 2}\right)^{-1}+O\left(\left(R^{k} k^{5 / 2}\right)^{-1}\right),
$$

где $\beta=0,4399237 \ldots$

Для доказательства полученных ниже результатов будет использована обобщенная схема размещения частиц по ячейкам, предложенная и подробно исследованная В. Ф. Колчиным [5]. Введем случайные величины $\eta_{1}, \ldots, \eta_{N}$, равные объемам деревьев леса из $F_{N, n}$ с номерами $1, \ldots, N$ соответственно. Пусть $\xi_{1}, \ldots, \xi_{N}$ - независимые одинаково распределенные целочисленные случайные величины такие, что

$$
p_{k}=\mathbf{P}\left\{\xi_{1}=k\right\}=\frac{T_{k+1} \lambda^{k+1}}{T(\lambda)}, \quad k=0,1, \ldots, \quad 0<\lambda \leqslant R .
$$

Легко проверить, что для целых неотрицательных $k_{1}, \ldots, k_{N}$

$$
\mathbf{P}\left\{\eta_{1}=k_{1}, \ldots, \eta_{N}=k_{N}\right\}=\mathbf{P}\left\{\xi_{1}=k_{1}, \ldots, \xi_{N}=k_{N} \mid \xi_{1}+\ldots+\xi_{N}=n\right\},
$$

а это и означает, что для двух наборов случайных величин $\left(\eta_{1}, \ldots, \eta_{N}\right)$ и $\left(\xi_{1}, \ldots, \xi_{N}\right)$ выполнены условия обобщенной схемы размещения [5].

Обозначим через $\eta_{(N)}$ и $\mu_{r}$, соответственно, максимальный объем дерева и число деревьев объема $r$ в лесе из $F_{N, n}$. Ниже будут использоваться два значения параметра $\lambda$ распределения (6). Если $N, n \rightarrow \infty$ так, что $N / \sqrt{n} \rightarrow \infty$, то в качестве $\lambda$ берем решение уравнения

$$
\frac{\lambda T^{\prime}(\lambda)}{T(\lambda)}=\frac{N+n}{N}
$$

а если $N, n \rightarrow \infty$ так, что $N / \sqrt{n} \leqslant C<\infty$, то полагаем $\lambda=R$. В случае $N / \sqrt{n} \rightarrow \infty$ обозначим

$$
m=\mathbf{E} \xi_{1}, \quad \sigma^{2}=\mathbf{D} \xi_{1} .
$$

При $\lambda=R$ из (1)-(3), (5) и (6) следует, что $E \xi_{1}$ и $\mathbf{D} \xi_{1}$ не существуют. Из (6) и (8) получаем, что

$$
m=\frac{\lambda T^{\prime}(\lambda)}{T(\lambda)}-1=\frac{n}{N}, \quad \sigma^{2}=\frac{\lambda^{2} T^{\prime \prime}(\lambda)}{T(\lambda)}-m-m^{2}
$$

Положим

$$
\sigma_{r r}^{2}=p_{r}\left(1-p_{r}-\frac{(m-r)^{2}}{\sigma^{2}} p_{r}\right)
$$

Справедливы следующие результаты.

Теорема 1. Пусть $N, n \rightarrow \infty$ так, что $n / N \rightarrow 0$, a $r$ выбрано так, что

$$
\frac{N}{r^{3 / 2}}\left(\frac{\lambda}{r}\right)^{r} \rightarrow \infty, \quad N T_{r+2} \lambda^{r+1} \rightarrow \gamma,
$$

где $\gamma$ - некоторая неотрицательная постоянная. Тогда

$$
\mathbf{P}\left\{\eta_{(N)}=r\right\} \rightarrow e^{-\gamma}, \quad \mathbf{P}\left\{\eta_{(N)}=r+1\right\} \rightarrow 1-e^{-\gamma} .
$$


Теорема 2. Пусть $N, n \rightarrow \infty$ так, что $n / N \rightarrow c$, a $r$ выбрано так, что

$$
\frac{N \beta}{r^{3 / 2}}\left(\frac{\lambda}{R}\right)^{r+1} \rightarrow \gamma
$$

где с, $\gamma$ - некоторые положительные постоянные. Тогда для любого фиксированного $k=0, \pm 1, \pm 2, \ldots$

$$
\mathbf{P}\left\{\eta_{(N)} \leqslant r+k\right\} \rightarrow \exp \left\{-\gamma\left(\lambda_{c} / R\right)^{k+1}\left(1-\lambda_{c} / R\right)^{-1}\right\},
$$

где $\lambda_{c}$ удовлетворяет равенству

$$
\frac{\lambda_{c} T^{\prime}\left(\lambda_{c}\right)}{T\left(\lambda_{c}\right)}=1+c
$$

Теорема 3. Пусть $N, n \rightarrow \infty$ так, что $n / N \rightarrow \infty, N / \sqrt{n} \rightarrow \infty$. Тогда для любого фиксированного z

$$
\mathbf{P}\left\{\ln (R / \lambda) \eta_{(N)}-u \leqslant z\right\} \rightarrow e^{-e^{-z}}
$$

где и выбрано так, что

$$
N \beta \sqrt{\ln (R / \lambda)} u^{-3 / 2} e^{-u}=1
$$

Теорема 4. Пусть $N, n \rightarrow \infty$ так, что $2 n /\left(b^{2} R N^{2}\right) \rightarrow \gamma$, где $\gamma-$ некоторая положительная постоянная. Тогда для любого фиксированного $z>0$

$$
\mathbf{P}\left\{\eta_{(N)} / n \leqslant z\right\} \rightarrow \gamma^{3 / 2} \exp \left\{\frac{1}{2 \gamma}\right\} \sum_{s=0}^{\infty} \frac{(-1)^{s}}{s !} I_{s}(z \gamma, \gamma)
$$

zde

$$
\begin{aligned}
I_{0}(u, v) & =\left(v^{3} \exp \{1 / v\}\right)^{-1 / 2} \\
I_{s}(u, v) & =\int_{X_{s}(u, v)} \frac{\exp \left\{-1 /\left(2\left(v-x_{1}-\ldots-x_{s}\right)\right)\right\}}{(2 \pi)^{s / 2}\left(x_{1} \ldots x_{s}\left(v-x_{1}-\ldots-x_{s}\right)\right)^{3 / 2}} d x_{1} \ldots d x_{s} \\
X_{s}(u, v) & =\left\{x_{i} \geqslant u, i=1, \ldots, s, x_{1}+\ldots+x_{s} \leqslant v\right\}, \quad s=1,2, \ldots
\end{aligned}
$$

Теорема 5. Пусть $N, n \rightarrow \infty$ так, что $N / \sqrt{n} \rightarrow 0$. Тогда для любого фиксированного $z>0$

$$
\mathbf{P}\left\{\frac{2\left(n-\eta_{(N)}\right)}{b^{2} R N^{2}} \leqslant z\right\} \rightarrow \frac{1}{\sqrt{2 \pi}} \int_{0}^{z} y^{-3 / 2} e^{-1 /(2 y)} d y .
$$

Теорема 6. Пусть $N, n \rightarrow \infty$ так, что $n / N \rightarrow 0$. Тогда для любого фиксированного $r \geqslant 2$ и чельх неотричательных $k$

$$
\mathbf{P}\left\{\mu_{r}=k\right\}=\frac{1}{k !}\left(N p_{r}\right)^{k} \exp \left\{-N p_{r}\right\}(1+o(1))
$$

равномерно относительно $\left(k-N p_{r}\right) / \sqrt{N p_{r}}$ в любом фиксированном конечном интервале. 
Теорема 7. Пусть $N, n \rightarrow \infty$ так, что $N / \sqrt{n} \rightarrow \infty$, ar $\geqslant 2$ фиксировано и $N \lambda^{r} \rightarrow \infty$. Тогда для иелых неотрицательных $k$

$$
\mathbf{P}\left\{\mu_{r}=k\right\}=\left(\sigma_{r r} \sqrt{2 \pi N}\right)^{-1} e^{-u_{r}^{2} / 2}(1+o(1))
$$

равномерно относительно $u_{r}=\left(k-N p_{r}\right) /\left(\sigma_{r r} \sqrt{N}\right)$ в любом фиксированном конечном интервале.

Это утверждение сохраняет силу, если $r=0, n^{2} / N \rightarrow \infty$, и если $r=1, n^{3} / N^{2} \rightarrow \infty$.

Теорема 8. Пусть $N, n \rightarrow \infty$ так, что $N / \sqrt{n} \leqslant C<\infty$. Тогда для фиксированного $r$ и иельх неотрицательных $k$

$$
\mathbf{P}\left\{\mu_{r}=k\right\}=\left(2 \pi N p_{r}\left(1-p_{r}\right)\right)^{-1 / 2} e^{-u_{r}^{2} / 2}(1+o(1))
$$

равномерно относительно $u_{r}=\left(k-N p_{r}\right) / \sqrt{N p_{r}\left(1-p_{r}\right)}$ в любом фиксированном конечном интервале.

Теорема 9. Пусть $N, n, r \rightarrow \infty$. Тогда для цельх неотричательных $k$

$$
\mathbf{P}\left\{\mu_{r}=k\right\}=\frac{1}{k !}\left(N p_{r}\right)^{k} \exp \left\{-N p_{r}\right\}(1+o(1))
$$

равномерно относительно $\left(k-N p_{r}\right) / \sqrt{N p_{r}}$ в любом фиксированном конечном интервале.

Сравнив теоремы 1-9 с результатами работ $[1,2,3]$, легко убедиться, что предельные распределения рассматриваемых характеристик случайных лесов с помеченными и непомеченными вершинами отличаются только значениями параметров. Из теоремы 5 следует также, что при $N / \sqrt{n} \rightarrow 0$ в случайном непомеченном лесе возникает гигантское дерево в смысле определення, данного в [6], это дерево имеет объем $n+o(n)$.

Пусть $r$ - целое неотрицательное число. Введем независимые вспомогательные случайные величины $\xi_{i}^{(j)}=\xi_{i}^{(j)}(r), i=1, \ldots, N, j=1,2$, такие, что

$$
\begin{aligned}
& \mathbf{P}\left\{\xi_{i}^{(1)}=k\right\}=\mathbf{P}\left\{\xi_{1}=k \mid \xi_{1} \leqslant r\right\}, \\
& \mathbf{P}\left\{\xi_{i}^{(2)}=k\right\}=\mathbf{P}\left\{\xi_{1}=k \mid \xi_{1} \neq r\right\} .
\end{aligned}
$$

Положим

$$
\zeta_{N}=\xi_{1}+\ldots+\xi_{N}, \quad \zeta_{N}^{(j)}=\xi_{1}^{(j)}+\ldots+\xi_{N}^{(j)}, \quad j=1,2, \quad P_{r}=\mathbf{P}\left\{\xi_{1}>r\right\} .
$$

Для доказательства теорем 1-9 удобно использовать следующие леммы 1 и 2, справедливые, как показано в [5], в условиях обобщенной схемы размещения и являющиеся следствиями соотношения (7).

Лемма 1. Справедливо равенство

$$
\mathbf{P}\left\{\eta_{(N)} \leqslant r\right\}=\left(1-P_{r}\right)^{N} \frac{\mathbf{P}\left\{\zeta_{N}^{(1)}=n\right\}}{\mathbf{P}\left\{\zeta_{N}=n\right\}}
$$

Лемма 2. Справедливо равенство

$$
\mathbf{P}\left\{\mu_{r}=k\right\}=\left(\begin{array}{l}
N \\
k
\end{array}\right) p_{r}^{k}\left(1-p_{r}\right)^{N-k} \frac{\mathbf{P}\left\{\zeta_{N-k}^{(2)}=n-k r\right\}}{\mathbf{P}\left\{\zeta_{N}=n\right\}} .
$$


Леммы 1 и 2 показывают, что для получения перечисленных выше результатов достаточно изучить предельное поведение бинома $\left(1-P_{r}\right)^{N}$, сумм независимых случайных величин $\zeta_{N}, \zeta_{N}^{(j)}, j=1,2$, и биномиальных вероятностей $\left(\begin{array}{c}N \\ k\end{array}\right) p_{r}^{k}\left(1-p_{r}\right)^{N-k}$. Ниже приводятся вспомогательные утверждения (леммы 3-11) об асимптотике указанных величин, а затем с их помощью доказываются теоремы 1-9.

Лемма 3. Пусть выполнены условия теоремы 1. Тогда

$$
N P_{r-1} \rightarrow \infty, \quad N P_{r} \rightarrow \gamma, \quad N P_{r+1} \rightarrow 0 .
$$

Доказательство. Из (2) и (8) следует, что $\lambda \rightarrow 0$, поэтому из (1) и (6) находим, что

$$
P_{r}=T_{r+2} \lambda^{r+1}(1+o(1)) \text {. }
$$

Отсюда непосредственно следуют утверждения леммы 3.

Лемма 4. Если выполнены условия теоремы 2, то при любом фиксированном $k$

$$
N P_{r+k} \rightarrow \gamma\left(\lambda_{c} / R\right)^{k+1}\left(1-\lambda_{c} / R\right)^{-1} .
$$

Доказательство. Очевидно, что

$$
N P_{r+k}=N \mathbf{P}\left\{\xi_{1}=r\right\} \sum_{s=1}^{\infty} \mathbf{P}\left\{\xi_{1}=r+k+s\right\} / \mathbf{P}\left\{\xi_{1}=r\right\}
$$

Нетрудно видеть, что $r \rightarrow \infty$, поэтому из (5) и (6) следует, что

$$
\begin{gathered}
\frac{\mathbf{P}\left\{\xi_{1}=r+k+s\right\}}{\mathbf{P}\left\{\xi_{1}=r\right\}}=\frac{T_{r+k+s+1} \lambda^{k+s}}{T_{r+1}}=(\lambda / R)^{k+s}(1+O(s / r)), \\
\mathbf{P}\left\{\xi_{1}=r\right\}=\frac{\beta}{r^{3 / 2}}\left(\frac{\lambda}{R}\right)^{r+1}(1+o(1)) .
\end{gathered}
$$

Из этих соотношений и (8) следует утверждение леммы 4.

Лемма 5. Пусть $N, n \rightarrow \infty$ так, что

$$
n / N \rightarrow \infty, \quad N / \sqrt{n} \rightarrow \infty, \quad r=(u+z) / \ln (R / \lambda)+O(1),
$$

где z-фиксированное число, а и задано соотночением (11). Тогда

$$
N P_{r} \rightarrow e^{-z} \text {. }
$$

Доказательство. Из (1)-(3) и (8) следует, что в условиях леммы $\lambda \rightarrow R$. Из (3), (5) и (6) получаем соотношение

$$
P_{r}=\beta(1+o(1)) \sum_{s=1}^{\infty}(r+s)^{-3 / 2} \exp \{-(r+s) \ln (R / \lambda)\} .
$$

Нетрудно видеть, что

$$
\begin{aligned}
\int_{1}^{\infty}(r+y)^{-3 / 2} \exp \{-(r+y) \ln (R / \lambda)\} d y & <\sum_{s=1}^{\infty}(r+s)^{-3 / 2} \exp \{-(r+s) \ln (R / \lambda)\} \\
& <\int_{0}^{\infty}(r+y)^{-3 / 2} \exp \{-(r+y) \ln (R / \lambda)\} d y,
\end{aligned}
$$


поэтому

$$
\begin{aligned}
P_{r}= & \beta(1+o(1)) \int_{r}^{\infty} y^{-3 / 2} \exp \{-y \ln (R / \lambda)\} d y+O\left(\int_{r}^{r+1} y^{-3 / 2} \exp \{-y \ln (R / \lambda)\} d y\right) \\
=\beta & \sqrt{\ln (R / \lambda)}(1+o(1)) \\
& \times \int_{r \ln (R / \lambda)}^{\infty} y^{-3 / 2} e^{-y} d y+O\left(\sqrt{\ln (R / \lambda)} \int_{r \ln (R / \lambda)}^{(r+1) \ln (R / \lambda)} y^{-3 / 2} e^{-y} d y\right) .
\end{aligned}
$$

Из (4) и (8) следует, что

$$
\lambda=R-(b R N /(2 n))^{2}(1+o(1)),
$$

поэтому из (11) нетрудно получить, что в условиях леммы $u \rightarrow \infty$. Отсюда, из (14), (15) и соотношения

$$
\int_{x}^{\infty} y^{h} e^{-y} d y=x^{h} e^{-x}(1+o(1)),
$$

справедливого при $x \rightarrow \infty$ и фиксированном $h$, находим, что

$$
P_{r}=\beta \sqrt{\ln (R / \lambda)} u^{-3 / 2} e^{-(u+z)}(1+o(1)) .
$$

Отсюда и из (11) получаем, что $N P_{r} \rightarrow e^{-z}$.

Лемма 6. Пусть $N, n \rightarrow \infty$ так, что $N / \sqrt{n} \rightarrow 0 u r=n+o(n)$. Тогда $N P_{r} \rightarrow 0$.

Доказательство. Из (3), (5) и (6) следует, что

$$
P_{r}=\beta(1+o(1)) \sum_{s=1}^{\infty}(r+s+1)^{-3 / 2}
$$

Полагая $y=(r+s+1) / N^{2}$ и заменяя суммирование интегрированием, получаем, что

$$
N P_{r}=\beta \int_{A}^{\infty} y^{-3 / 2} d y+\varepsilon
$$

где выбором достаточно больших $N, n$ величину $A$ можно сделать сколь угодно большой, а $\varepsilon$ сколь угодно малой. Отсюда следует утверждение леммы 6.

Для нахождения асимптотики вероятностей, входящих в правые части утверждений лемм 1 и 2, нужно установить локальную сходимость распределений сумм $\zeta_{N}, \zeta_{N}^{(j)}$, $j=1,2$, к предельным законам. Однако, из (8) видно, что при $N / \sqrt{n} \rightarrow \infty$ слагаемые в этих суммах образуют схемы серий. Для сумм такого вида, встречающихся при использовании обобщенной схемы размещения, во многих случаях справедливы доказанные в $[8,9,10]$ локальные предельные теоремы. Ниже, в леммах 7-9, приведены некоторые результаты этих работ, которые будут полезны далее при доказательстве теорем.

Пусть $v_{1}, v_{2}, \ldots$ - независимые одинаково распределенные целочисленные неотрицательные случайные величины такие, что

$$
\mathbf{P}\left\{v_{1}=k\right\}=\frac{d_{k} \theta^{k}}{k ! B(\theta)}, \quad k=0,1,2, \ldots,
$$


где $d_{0}, d_{1}>0$, а ряд

$$
B(\theta)=\sum_{k=0}^{\infty} \frac{d_{k} \theta^{k}}{k !}
$$

имеет радиус сходимости $H>0$. Нетрудно видеть, что

$$
\begin{aligned}
a & =\mathbf{E} v_{1}=\frac{\theta B^{\prime}(\theta)}{B(\theta)}, \\
D^{2} & =\mathbf{D} v_{1}=\frac{\theta^{2} B^{\prime \prime}(\theta)}{B(\theta)}+\frac{\theta B^{\prime}(\theta)}{B(\theta)}-\frac{\theta^{2}\left(B^{\prime}(\theta)\right)^{2}}{(B(\theta))^{2}} .
\end{aligned}
$$

Введем обозначение

$$
P_{N}(n)=\mathbf{P}\left\{v_{1}+\ldots+v_{N}=n\right\} .
$$

Лемма 7. Пусть $N \rightarrow \infty, \theta=\theta(N) \rightarrow 0, \theta N \rightarrow \infty$. Тогда

$$
D \sqrt{N} P_{N}(n)-\frac{1}{\sqrt{2 \pi}} \exp \left\{-\frac{(n-a N)^{2}}{2 D^{2} N}\right\} \rightarrow 0
$$

равномерно относительно челых неотричательных $n$.

Лемма 8. Пусть $N \rightarrow \infty$ и существуют положительные постоянные $\theta_{0}, \theta_{1}$ такие, что параметр $\theta=\theta(N)$ меняется так, что $\theta_{0} \leqslant \theta \leqslant \theta_{1}<H$. Тогда

$$
D \sqrt{N} P_{N}(n)-\frac{1}{\sqrt{2 \pi}} \exp \left\{-\frac{(n-a N)^{2}}{2 D^{2} N}\right\} \rightarrow 0
$$

равномерно относительно челых неотричательных $n$.

Замечание 1. Нетрудно видеть, что если $d_{0}=0, d_{1}>0, d_{2}>0$, то леммы 7 и 8 сохраняют силу. Для того, чтобы в этом убедиться, достаточно случайные величины $v_{1}, v_{2}, \ldots$ заменить на $v_{1}-1, v_{2}-1, \ldots$, а $a$ на $a-1$.

Замечание 2. В [9] показано, что леммы 7 и 8 сохраняют силу, если $d_{0}>0, d_{1}=0$, $d_{2}>0, \theta^{3} N \rightarrow \infty$.

В следующей лемме мы будем предполагать, что $d_{0}>0, d_{1} \geqslant 0$, а максимальный шаг распределения (17) равен единице.

Лемма 9. Пусть $N \rightarrow \infty$ и при $k \rightarrow \infty$ справедливо соотношение

$$
\mathbf{P}\left\{v_{1}=k\right\}=C k^{-\delta}(1+o(1)),
$$

где $C$ - некоторая положительная постоянная, $1<\delta<3$, а $\theta=\theta(N) \rightarrow H$ так, что

$$
\mathbf{E}\left(v_{1}-a\right)^{4} / D^{4} N \rightarrow 0 .
$$

Тогда

$$
D \sqrt{N} P_{N}(n)-\frac{1}{\sqrt{2 \pi}} \exp \left\{-\frac{(n-a N)^{2}}{2 D^{2} N}\right\} \rightarrow 0
$$

равномерно относительно иелых неотричательных $n$. 
В случае, когда $N / \sqrt{n} \rightarrow 0$, мы полагаем $\lambda=R$, следовательно, при оценке вероятности $\mathbf{P}\left\{\zeta_{N}=n\right\}$, а также вероятности $\mathbf{P}\left\{\zeta_{N}^{(1)}=n\right\}$ (см. леммы 1 и 2) в случае фиксированного $r$ схема серий не возникает, и можно использовать следующий результат из [11].

Лемма 10. Пусть $\nu_{1}, \nu_{2}, \ldots$ - последовательность независимых одинаково распределенных челочисленных случайных величин, имеючих функчию распределения, принадлежашую области притяжения устойчивого закона с плотностью распределения $g(x) u$ показателем $\alpha, \alpha \neq 1,2$. Пусть при $k \rightarrow \infty$

$$
\mathbf{P}\left\{v_{1}=k\right\}=C k^{-(1+\alpha)}(1+o(1)),
$$

где C - некоторая положительная постоянная. Тогда при $n N^{-1 / \alpha} \rightarrow \infty$

$$
P_{N}(n)=N^{-1 / \alpha} g\left(n N^{-1 / \alpha}\right)(1+o(1)) \text {. }
$$

При $r \rightarrow \infty$ слагаемые, входящие в суммы $\zeta_{N}^{(1)}$ и $\zeta_{N-k}^{(2)}$, образуют схемы серий, поэтому для оценки соответствующих вероятностей докажем следующую лемму.

Лемма 11. Пусть $N, n \rightarrow \infty$ так, что $N / \sqrt{n} \rightarrow 0$. Тогда справедливы следуючие утверждения.

(1) Если $r=n-z b^{2} R N^{2} / 2$, где $z-$ некоторая положительная постоянная, то

$$
\mathbf{P}\left\{\zeta_{N}^{(1)}=n\right\}=\frac{N \beta}{\sqrt{2 \pi} n^{3 / 2}} \int_{z}^{\infty} y^{-3 / 2} e^{-1 /(2 y)} d y(1+o(1)) .
$$

(2) Если $r \rightarrow \infty, S=N\left(1-p_{r}+o\left(p_{r}\right)\right), z=2 n /\left(b^{2} R N^{2}\right)$, mo

$$
\frac{b^{2} R N^{2}}{2} \mathbf{P}\left\{\frac{2 \zeta_{S}^{(2)}}{b^{2} R N^{2}}=z\right\}=\frac{1+o(1)}{\sqrt{2 \pi z^{3}}}
$$

Доказательство. Мы будем доказывать оба утверждения леммы 11 одновременно. Для этого обозначим рассматриваемые суммы через $\zeta_{S}^{(j)}$, где $S=N$ при $j=1$, а при $j=2$ значение $S$ указано в утверждении 2 . Символами $C_{1}, C_{2}, \ldots$ будем обозначать некоторые положительные постоянные.

Представим вероятность $\mathbf{P}\left\{\zeta_{S}^{(j)}=n\right\}$ в виде суммы

$$
\mathbf{P}\left\{\zeta_{S}^{(j)}=n\right\}=P_{1}^{(j)}+S P_{2}^{(j)}+P_{3}^{(j)}
$$

где

$$
\begin{aligned}
P_{1}^{(j)} & =\mathbf{P}\left\{\zeta_{S}^{(j)}=n, \xi_{i}^{(j)} \leqslant \gamma n, i=1, \ldots, S\right\}, \\
P_{2}^{(j)} & =\mathbf{P}\left\{\zeta_{S}^{(j)}=n, \xi_{i}^{(j)} \leqslant \gamma n, i=1, \ldots, S-1, \xi_{N}^{(j)}>\gamma n\right\}, \\
P_{3}^{(j)} & =\mathbf{P}\left\{\zeta_{S}^{(j)}=n, \bigcup_{i \neq k}\left(\xi_{i}^{(j)}>\gamma n, \xi_{k}^{(j)}>\gamma n\right)\right\}, \\
\gamma & =\left(S^{2} / n\right)^{1 / 6} .
\end{aligned}
$$


Оценивая последовательно вероятности $P_{1}^{(j)}, P_{2}^{(j)}, P_{3}^{(j)}$, мы увидим ниже, что основной вклад в сумму (18) дает второе слагаемое.

Рассмотрим $P_{1}^{(j)}$. Положим

$$
Q(\omega)=\sum_{k \leqslant \gamma n} \exp \{\omega k\} \mathbf{P}\left\{\xi_{1}^{(j)}=k\right\}
$$

Из (5), (6), (12), (13) и равенства $\lambda=R$ нетрудно получить следующее соотношение, справедливое при достаточно больших $l$ :

$$
\sum_{k \leqslant l} k \mathbf{P}\left\{\xi_{1}^{(j)}=k\right\} \leqslant C_{1} \sum_{k \leqslant l} k^{-1 / 2} \leqslant C_{2} \sqrt{l}
$$

Аналогично,

$$
\sum_{k>l} \mathbf{P}\left\{\xi_{1}^{(j)}=k\right\} \leqslant C_{3} \int_{l}^{\infty} y^{-3 / 2} d y=2 C_{3} l^{-1 / 2}
$$

Поскольку $S / \sqrt{n} \rightarrow 0$, из (19) следует, что

$$
(\gamma n)^{-1 / 2}=o\left(S^{-1}\right)
$$

Отсюда и из (20)-(22) получаем соотношение

$$
Q(1 /(\gamma n))=1+o\left(S^{-1}\right) .
$$

Введем вспомогательные независимые одинаково распределенные случайные величины $\tilde{\xi}_{1}^{(j)}, \ldots, \tilde{\xi}_{S}^{(j)}$, имеющие следующее распределение вероятностей:

$$
\mathbf{P}\left\{\tilde{\xi}_{1}^{(j)}=k\right\}=\exp \{k /(\gamma n)\} \mathbf{P}\left\{\xi_{1}^{(j)}=k\right\} / Q(1 /(\gamma n)),
$$

где $k \leqslant \gamma n$. Положим

$$
\tilde{\zeta}_{S}^{(j)}=\tilde{\xi}_{1}^{(j)}+\ldots+\tilde{\xi}_{S}^{(j)}
$$

Легко видеть, что

$$
P_{1}^{(j)}=Q^{S}(1 /(\gamma n)) e^{-1 / \gamma} \mathbf{P}\left\{\tilde{\zeta}_{S}^{(j)}=n\right\}
$$

Докажем, что при достаточно больших $n$

$$
\mathbf{P}\left\{\tilde{\zeta}_{S}^{(j)}=n\right\} \leqslant C_{4} S^{-2}
$$

Обозначим через $\tilde{\varphi}_{j}(t)$ характеристическую функцию случайной величины $\tilde{\xi}_{1}^{(j)}$. По формуле обращения

$$
\mathbf{P}\left\{\tilde{\zeta}_{S}^{(j)}=n\right\}=\frac{1}{\pi b^{2} R S^{2}} \int_{-\pi b^{2} R S^{2} / 2}^{\pi b^{2} R S^{2} / 2} e^{-i t v}\left(\tilde{\varphi}_{j}\left(\frac{2 t}{b^{2} R S^{2}}\right)\right)^{S} d t
$$

где $v=2 n /\left(b^{2} R S^{2}\right)$. Рассмотрим выражение

$$
\left|\tilde{\varphi}_{j}(t)\right|^{S}=\left|\frac{Q(1 /(\gamma n)+i t)}{Q(1 /(\gamma n))}\right|^{S} .
$$


Если $k \leqslant \gamma n$, то

$$
\exp \left\{\frac{k}{\gamma n}\right\} \leqslant 1+\frac{2 k}{\gamma n}
$$

поэтому из (20), (21) и (23) нетрудно получить, что

$$
\left|Q\left(\frac{1}{\gamma n}+i t\right)\right| \leqslant\left|\tilde{\varphi}_{j}(t)\right|+\sum_{k>\gamma n} \mathbf{P}\left\{\xi_{1}^{(j)}=k\right\}+o\left(S^{-1}\right) .
$$

Отсюда и из (22), (23) следует неравенство

$$
\left|Q\left(\frac{1}{\gamma n}+i t\right)\right| \leqslant\left|\tilde{\varphi}_{j}(t)\right|+o\left(S^{-1}\right) .
$$

Обозначим через $\varphi(t), \varphi_{1}(t)$ и $\varphi_{2}(t)$, соответственно, характеристические функции случайных величин $\xi_{1}, \xi_{1}^{(1)}, \xi_{1}^{(2)}$. Используя (1), (3), (6), нетрудно получить, что

$$
\begin{aligned}
\varphi(t) & =T\left(R e^{i t}\right) / e^{i t} \\
\varphi_{1}(t) & =\frac{\varphi(t)-\sum_{k=r+1}^{\infty} e^{i t k} p_{k}}{1-P_{r}} \\
\varphi_{2}(t) & =\frac{\varphi(t)-e^{i t r} p_{r}}{1-p_{r}}
\end{aligned}
$$

Используя эти равенства, несложно показать, что при любом фиксированном $t$ справедливы неравенства $\left|\varphi_{j}(t)\right| \geqslant C_{5}, j=1,2$, поэтому из (24), (28) и (29) вытекает, что

$$
\left|\tilde{\varphi}_{j}(t)\right|^{S} \leqslant C_{6}\left|\varphi_{j}(t)\right|^{S}
$$

Учитывая (4) и (30), получаем, что при $t \rightarrow 0$

$$
\begin{aligned}
\varphi(t) & =\exp \{-b \sqrt{-R i t}+o(\sqrt{t})\} \\
& =\exp \{-b \sqrt{R|t| / 2}(1-i t /|t|)+o(\sqrt{t})\}
\end{aligned}
$$

Отсюда находим, что при $|t| \leqslant \varepsilon S^{2}$ и достаточно малом $\varepsilon$

$$
\left|\varphi\left(2 t /\left(b^{2} R S^{2}\right)\right)\right| \leqslant \exp \left\{-C_{7} \sqrt{|t|} / S\right\} .
$$

Неравенства (22), (30) и (33) при $j=1$ приводят к оценке

$$
\left|\varphi_{1}\left(2 t /\left(b^{2} R S^{2}\right)\right)\right|^{S} \leqslant C_{8} \exp \left\{-C_{9} \sqrt{|t|}\right\} .
$$

В [7] показано, что если

$$
2|t| r^{2} /\left(b^{4} R^{2}\right) \leqslant 1
$$

To

$$
\left|\varphi_{2}\left(2 t /\left(b^{2} R\right)\right)\right| \leqslant\left|\varphi\left(2 t /\left(b^{2} R\right)\right)\right|^{1 / 2},
$$


а если

$$
b^{4} R^{2} N^{2} /\left(2 r^{2}\right)<|t| \leqslant \varepsilon N^{2},
$$

то при достаточно больших $N$ и достаточно малом $\varepsilon$

$$
\left|\varphi_{2}\left(\frac{2 t}{b^{2} R N^{2}}\right)\right|^{S} \leqslant \exp \left\{S\left(-\frac{C_{10} \sqrt{|t|}}{N}+\frac{C_{11}}{r^{3 / 2}}\right)\right\} .
$$

Если $\varepsilon<|t| \leqslant \pi$, то, как хорошо известно, $|\varphi(t)| \leqslant e^{-C_{12}}$, поэтому из (30) несложно получить, учитывая соотношение $r \rightarrow \infty$, что при $\varepsilon S^{2}<|t| \leqslant \pi b^{2} R S^{2} / 2$

$$
\left|\varphi_{j}\left(2 t /\left(b^{2} R S^{2}\right)\right)\right|^{S} \leqslant e^{-C_{13}} .
$$

Представим интеграл, стоящий в правой части (27), в виде суммы двух интегралов, области интегрирования которых определяются неравенствами $|t| \leqslant \varepsilon S^{2}$ и $\varepsilon S^{2}<|t| \leqslant \pi b^{2} R S^{2} / 2$, причем для $j=2$ область интегрирования первого интеграла можно разбить на две части в соответствии с (35) и (37). Тогда, используя (34), (39), (36) и (38), получаем (26). Из (19), (24)-(26) следует, что

$$
P_{1}^{(j)} \leqslant C_{14} S^{-2} e^{-1 / \gamma}=o\left(S / n^{3 / 2}\right) .
$$

Оценим $P_{2}^{(j)}$. Очевидно, что

$$
\begin{aligned}
P_{2}^{(j)}=\sum_{n-q_{j} \leqslant k \leqslant n(1-\gamma)} & \mathbf{P}\left\{\xi_{S}^{(j)}=n-k\right\} \\
& \times \mathbf{P}\left\{\xi_{1}^{(j)}+\ldots+\xi_{S-1}^{(j)}=k, \xi_{i}^{(j)} \leqslant \gamma n, i=1, \ldots, S-1\right\},
\end{aligned}
$$

где $q_{1}=r, q_{2}=n$.

Введем вспомогательные случайные величины $\xi_{i}^{(j)}(\gamma n), i=1, \ldots, S, j=1,2$, такие, что

$$
\begin{aligned}
& \mathbf{P}\left\{\xi_{i}^{(1)}(\gamma n)=k\right\}=\mathbf{P}\left\{\xi_{i}=k \mid \xi_{i} \leqslant \gamma n\right\}, \\
& \mathbf{P}\left\{\xi_{i}^{(2)}(\gamma n)=k\right\}=\mathbf{P}\left\{\xi_{i}=k \mid \xi_{i} \leqslant \gamma n, \xi_{i} \neq r\right\},
\end{aligned}
$$

пусть, кроме того,

$$
\zeta_{S}^{(j)}(\gamma n)=\xi_{1}^{(j)}(\gamma n)+\ldots+\xi_{S}^{(j)}(\gamma n) .
$$

Используя (22), (41) и (42), нетрудно получить, что

$$
P_{2}^{(j)}=(1+o(1)) \sum_{n-q_{i} \leqslant k<n(1-\gamma)} \mathbf{P}\left\{\xi_{S}^{(j)}=n-k\right\} \mathbf{P}\left\{\zeta_{S-1}^{(j)}(\gamma n)=k\right\}
$$

Обозначим через $\varphi^{(\gamma n)}(t)$ характеристическую функщию случайной величины $\xi_{1}^{(1)}(\gamma n)$. Используя (6), (19), (22), аналогично (32), находим, что при любом фиксированном $t$

$$
\left(\varphi^{(\gamma n)}\left(2 t /\left(b^{2} R S^{2}\right)\right)\right)^{S-1} \rightarrow \exp \{-\sqrt{|t|}(1-i t /|t|)\} .
$$


Последнее выражение, как известно (см., например, [5]), является характеристической функщей устойчивого закона с показателем $\alpha=1 / 2$, имеющего плотность $\left(2 \pi x^{3} \exp \{1 / x\}\right)^{-1 / 2}$. Отсюда и из (44) следует, что

$$
\mathbf{P}\left\{\zeta_{S-1}^{(1)}(\gamma n) \leqslant y b^{2} R S^{2} / 2\right\} \rightarrow \frac{1}{\sqrt{2 \pi}} \int_{0}^{y} x^{-3 / 2} e^{-1 /(2 x)} d x .
$$

Покажем, что при достаточно больших $n$ и $j=1,2$

$$
\mathbf{P}\left\{\zeta_{S-1}^{(j)}(\gamma n)>\gamma^{-1} N^{2}\right\} \leqslant C_{15} \gamma^{1 / 2}
$$

Легко видеть, что

$$
\begin{aligned}
\mathbf{P}\left\{\zeta_{S-1}^{(j)}(\gamma n)>\gamma^{-1} N^{2}\right\} \\
\quad \leqslant(S-1) \mathbf{P}\left\{\xi_{1}^{(j)}>\gamma^{-1} N^{2}\right\}+G_{j}^{S-1} \mathbf{P}\left\{\zeta_{S-1}^{(j)}\left(\gamma^{-1} N^{2}\right)>\gamma^{-1} N^{2}\right\},
\end{aligned}
$$

где, в соответствии с (42), $\gamma^{-1} N^{2}$ стоит вместо $\gamma n, G_{1}=\left(1-P_{\gamma^{-1}} N^{2}\right) /\left(1-P_{\gamma n}\right), G_{2}=1$. Заметим, что

$$
\mathbf{P}\left\{\xi_{1}^{(j)}(\gamma n)>\gamma^{-1} N^{2}\right\}=\sum_{k>\gamma^{-1} N^{2}} \mathbf{P}\left\{\xi_{1}^{(j)}(\gamma n)=k\right\} \leqslant C_{16} \mathbf{P}\left\{\xi_{1}^{(j)}>\gamma^{-1} N^{2}\right\} .
$$

Отсюда и из (22) получаем неравенство

$$
(S-1) \mathbf{P}\left\{\xi_{1}^{(j)}(\gamma n)>\gamma^{-1} N^{2}\right\} \leqslant C_{17} \gamma^{1 / 2}
$$

С помощью (21) находим оценку

$$
\mathbf{E} \xi_{1}^{(j)}\left(\gamma^{-1} N^{2}\right) \leqslant C_{18} \sum_{k<\gamma^{-1} N^{2}} k \mathbf{P}\left\{\xi_{1}=k\right\} \leqslant C_{19} \gamma^{-1 / 2}
$$

и, применяя неравенство Чебышёва, видим, что

$$
\mathbf{P}\left\{\zeta_{S-1}^{(j)}\left(\gamma^{-1} N^{2}\right)>\gamma^{-1} N^{2}\right\} \leqslant C_{20} \gamma^{1 / 2}
$$

В силу (22) справедливо соотношение $G_{1}^{S-1}=1+o(1)$, поэтому из (47)-(49) следует (46).

Учитывая (43), представим вероятность $P_{2}^{(j)}$ в виде суммы

$$
P_{2}^{(j)}=L_{1}^{(j)}+L_{2}^{(j)}+L_{3}^{(j)}
$$

где

$$
\begin{aligned}
L_{i}^{(j)} & =(1+o(1)) \sum_{K_{i}} \mathbf{P}\left\{\xi_{S}^{(j)}=n-k\right\} \mathbf{P}\left\{\zeta_{S-1}^{(j)}(\gamma n)=k\right\}, \quad i=1,2,3 \\
K_{1} & =\left\{k: n-q_{i} \leqslant k \leqslant \gamma^{-1} N^{2}\right\} \\
K_{2} & =\left\{k: \gamma^{-1} N^{2}<k \leqslant n\left(1-\gamma^{1 / 6}\right)\right\} \\
K_{3} & =\left\{k: n\left(1-\gamma^{1 / 6}\right)<k<n(1-\gamma)\right\} .
\end{aligned}
$$


Используя (5), (6), (12), (13) и (22), нетрудно получить, что при выполнении условий леммы

$$
L_{1}^{(j)}=\beta n^{-3 / 2}(1+o(1)) \mathbf{P}\left\{n-q_{j} \leqslant \zeta_{S-1}^{(j)}(\gamma n) \leqslant \gamma^{-1} N^{2}\right\} .
$$

Отсюда и из (45), (46) находим, полагая $j=1, q_{1}=r, r=n-z b^{2} R N^{2} / 2$, что

$$
L_{1}^{(1)}=\beta\left(2 \pi n^{3}\right)^{-1 / 2}(1+o(1)) \int_{z}^{\infty} y^{-3 / 2} e^{-1 /(2 y)} d y
$$

а в случае $j=2, q_{2}=n$ и $r \rightarrow \infty$

$$
L_{1}^{(2)}=\beta n^{-3 / 2}(1+o(1)) .
$$

Если $k \in K_{2}$, то из (5), (6), (12), (13) следует неравенство

$$
\mathbf{P}\left\{\xi_{S}^{(j)}=n-k\right\} \leqslant C_{21}\left(n \gamma^{1 / 6}\right)^{-3 / 2},
$$

поэтому из (46) вытекает, что

$$
L_{2}^{(j)} \leqslant C_{22}\left(n \gamma^{1 / 6}\right)^{-3 / 2} \mathbf{P}\left\{\zeta_{S-1}^{(j)}(\gamma n)>\gamma^{-1} N^{2}\right\}=o\left(n^{-3 / 2}\right) .
$$

Если $k \in K_{3}$, то, рассуждая аналогично, находим, что

$$
\mathbf{P}\left\{\xi_{S}^{(j)}=n-k\right\} \leqslant C_{23}(n \gamma)^{-3 / 2},
$$

откуда,

$$
L_{3}^{(j)} \leqslant C_{24}(n \gamma)^{-3 / 2} \mathbf{P}\left\{\zeta_{S-1}^{(j)}(\gamma n)>n\left(1-\gamma^{1 / 6}\right)\right\}
$$

Оценивая $\mathbf{E} \xi_{1}^{(j)}(\gamma n)$ и используя (21), (55) и неравенство Чебышёва, нетрудно получить соотношение

$$
\mathbf{P}\left\{\zeta_{S-1}^{(j)}(\gamma n)>n\left(1-\gamma^{1 / 6}\right)\right\} \leqslant C_{25} S(\gamma / n)^{1 / 2},
$$

поэтому из (55) следует, что

$$
L_{3}^{(j)} \leqslant C_{26} S /\left(n^{2} \gamma\right)=o\left(n^{-3 / 2}\right)
$$

Отсюда и из (50)-(53) видим, что

$$
P_{2}^{(j)}=L_{1}^{(j)}(1+o(1))
$$

Оценим, наконец, $P_{3}^{(j)}$. Легко заметить, что

$P_{3}^{(j)}=S(S-1) 2^{-1} \sum_{k<n(1-2 \gamma)} \mathbf{P}\left\{\zeta_{S-2}^{(j)}=k\right\} \mathbf{P}\left\{\xi_{S-1}^{(j)}+\xi_{S}^{(j)}=n-k, \xi_{S-1}^{(j)}>\gamma n, \xi_{S}^{(j)}>\gamma n\right\}$.

Отсюда следует, что

$$
P_{3}^{(j)} \leqslant C_{27} S^{2} \sum_{k<n(1-2 \gamma)} \mathbf{P}\left\{\zeta_{S-2}^{(j)}=k\right\} \sum_{K} \mathbf{P}\left\{\xi_{S-1}^{(j)}=s\right\} \mathbf{P}\left\{\xi_{S}^{(j)}=n-k-s\right\}
$$


где $K=\{s: \gamma n<s<n(1-\gamma)-k\}$. Используя (22) и (54), получаем, что

$$
\sum_{K} \mathbf{P}\left\{\xi_{S-1}^{(j)}=s\right\} \mathbf{P}\left\{\xi_{S}^{(j)}=n-k-s\right\} \leqslant C_{28}(\gamma n)^{-3 / 2} \mathbf{P}\left\{\xi_{S}^{(j)}>\gamma n\right\}<C_{29}(\gamma n)^{-2},
$$

и, в силу (57),

$$
P_{3}^{(j)} \leqslant C_{30} S^{2}(\gamma n)^{-2}=o\left(S / n^{3 / 2}\right) .
$$

Отсюда, из (18), (40), (51)-(53), (56) и известного (см., например, [4]) равенства

$$
\beta=b \sqrt{R} /(2 \sqrt{\pi})
$$

получаем оба утверждения леммы 11.

Теперь мы можем доказать сформулированные выше теоремы.

Доказательство теоремы 1. Из леммы 3 следует, что

$$
\left(1-P_{r-1}\right)^{N} \rightarrow 0, \quad\left(1-P_{r}\right)^{N} \rightarrow e^{-\gamma}, \quad\left(1-P_{r+1}\right)^{N} \rightarrow 1 .
$$

Положим

$$
m_{1}=\mathbf{E} \xi_{1}^{(1)}, \quad \sigma_{1}^{2}=\mathbf{D} \xi_{1}^{(1)} .
$$

Используя (6) и (12), нетрудно показать, что

$$
m_{1}=\frac{m-\sum_{k=r+1}^{\infty} k p_{k}}{1-P_{r}}, \quad \sigma_{1}^{2}=\frac{\sigma^{2}+m^{2}-\sum_{k=r+1}^{\infty} k^{2} p_{k}}{1-P_{r}}-m_{1}^{2},
$$

где $m$ и $\sigma^{2}$ определены в (9). Из (2) и (8) легко видеть, что

$$
\lambda=(n / N)(1+o(1)) \text {. }
$$

Отсюда и из (6), (9) следует, что для сумм $\zeta_{N}$ и $\zeta_{N}^{(1)}$ выполнены условия леммы 7 , поэтому

$$
\begin{aligned}
& \mathbf{P}\left\{\zeta_{N}^{(1)}=n\right\}=\frac{1+o(1)}{\sigma_{1} \sqrt{2 \pi N}} \exp \left\{-\frac{\left(n-m_{1} N\right)^{2}}{2 \sigma_{1}^{2} N}\right\}, \\
& \mathbf{P}\left\{\zeta_{N}=n\right\}=(\sigma \sqrt{2 \pi N})^{-1}(1+o(1)) .
\end{aligned}
$$

Из этих соотношений и (9), (60), (61) вытекает, как нетрудно проверить, что

$$
\mathbf{P}\left\{\zeta_{N}^{(1)}=n\right\} / \mathbf{P}\left\{\zeta_{N}=n\right\} \rightarrow 1
$$

Из леммы $1,(59)$ и (64) получаем, что

$$
\mathbf{P}\left\{\eta_{(N)} \leqslant r-1\right\} \rightarrow 0, \quad \mathbf{P}\left\{\eta_{(N)} \leqslant r\right\} \rightarrow e^{-\gamma}, \quad \mathbf{P}\left\{\eta_{(N)} \leqslant r+1\right\} \rightarrow 1,
$$

откуда и следует утверждение теоремы 1.

Доказательство теоремы 2. Из (8) нетрудно получить, что $0<C_{1} \leqslant \lambda \leqslant C_{2}<R$, поэтому для сумм $\zeta_{N}$ и $\zeta_{N}^{(1)}$ выполнены условия леммы 8 , из которой вытекают соотношения (62), (63). Используя (9), (60), легко обнаружить, что

$$
\left(n-m_{1} N\right)^{2} /\left(\sigma_{1}^{2} N\right)=O\left(r^{2} / N\right),
$$

а выбор $r$ обеспечивает выполнение соотношения $r^{2} / N \rightarrow 0$. Поэтому, принимая во внимание (60), из (62), (63) получим (64). Утверждение теоремы 2 следует из (64) и лемм 1, 4 . 
Доказательство теоремы 3. С помощью (3), (4), (6), (9) и (15) нетрудно установить, что

$$
\sigma^{2}=\frac{2}{b^{2} R^{3}}\left(\frac{n}{N}\right)^{3}, \quad \mathbf{E}\left(\xi_{1}-m\right)^{4}=O\left((n / N)^{7}\right) .
$$

Это значит, с учетом (5), что для суммы $\zeta_{N}$ выполнены условия леммы 9, поэтому справедливо соотношение (63). Заметим, что $N P_{r} \rightarrow e^{-z}$ в силу леммы 5. В [10] показано, что этого условия достаточно, чтобы лемма 9 была справедлива и для суммы $\zeta_{N}^{(1)}$, значит, равенство (62) имеет место и в этом случае. Используя (3), (4), (6), (9), (12) и (15), можно установить, что

$$
\sigma_{1}^{2}=\sigma^{2}(1+o(1))
$$

По аналогии с (16), нетрудно показать, что

$$
\sum_{k=r+1}^{\infty} k p_{k}=\beta \sqrt{\ln (R / \lambda)} u^{-1 / 2} e^{-(u+z)}(1+o(1)) .
$$

Отсюда и из соотношений $P_{r}=O\left(N^{-1}\right),(11),(16),(60),(65),(66)$ следует, что $\left(n-m_{1} N\right)^{2} /\left(\sigma_{1}^{2} N\right) \rightarrow 0$, поэтому из (62), (63) получаем (64). Для завершения доказательства осталось применить леммы 1 и 5 .

Теорема 4 доказана в [7].

Доказательство теоремы 5. Обозначим через $\psi(t)$ характеристическую функщию случайной величины $2 \zeta_{N} /\left(b^{2} R N^{2}\right)$. Из (32) следует, что

$$
\psi(t)=\varphi^{N}\left(2 t /\left(b^{2} R N^{2}\right)\right) \rightarrow \exp \{-\sqrt{-2 i t}\},
$$

а последнее выражение, как уже указывалось при доказательстве леммы 11 , является характеристической функцией устойчивого закона с показателем $\alpha=1 / 2$, имеющего плотность $\left(2 \pi x^{3} \exp \{1 / x\}\right)^{-1 / 2}$. Отсюда и из (3), (5), (6) получаем, что для суммы $\zeta_{N}$ выполнены условия леммы 10 , поэтому, учитывая (58), получаем, что

$$
\mathbf{P}\left\{\zeta_{N}=n\right\}=\frac{b \sqrt{R}}{2 \sqrt{\pi}} \frac{N}{n^{3 / 2}}(1+o(1))=\frac{\beta N}{n^{3 / 2}}(1+o(1)) .
$$

Отсюда и из лемм 1, 6, 11 очевидным образом следует утверждение теоремы 5.

Доказательство теоремы 6. Из условий теоремы находим, что

$$
k=N p_{r}+u \sqrt{N p_{r}}
$$

где $|u| \leqslant C<\infty$. Нетрудно видеть, что для суммы $\zeta_{N-k}^{(2)}$ выполнены условия леммы 7 , поэтому

$$
\mathbf{P}\left\{\zeta_{N-k}^{(2)}=n-k r\right\}=\frac{1+o(1)}{\sigma_{2} \sqrt{2 \pi(N-k)}} \exp \left\{-\frac{\left(n-k r-m_{2}(N-k)\right)^{2}}{2 \sigma_{2}^{2}(N-k)}\right\}
$$

где

$$
m_{2}=\mathbf{E} \xi_{1}^{(2)}=\frac{m-r p_{r}}{1-p_{r}}, \quad \sigma_{2}^{2}=\mathbf{D} \xi_{1}^{(2)}=\frac{\sigma^{2}}{\left(1-p_{r}\right)^{2}}\left(1-p_{r}-\frac{(m-r)^{2}}{\sigma^{2}} p_{r}\right) .
$$


Используя (2), (6), (9), (61), (69) и (71), нетрудно проверить, что

$$
\frac{\left(n-k r-m_{2}(N-k)\right)^{2}}{\sigma_{2}^{2}(N-k)} \rightarrow 0, \quad \sigma_{2}^{2}=\sigma^{2}(1+o(1)),
$$

поэтому из (63), (70) вытекает соотношение

$$
\mathbf{P}\left\{\zeta_{N-k}^{(2)}=n-k r\right\} / \mathbf{P}\left\{\zeta_{N}=n\right\} \rightarrow 1 .
$$

Поскольку $p_{r} \rightarrow 0$, для рассматриваемых значений $k$, как известно, можно использовать пуассоновское приближение биномиального распределения

$$
\left(\begin{array}{l}
N \\
k
\end{array}\right) p_{r}^{k}\left(1-p_{r}\right)^{N-k}=\frac{\left(N p_{r}\right)^{k}}{k !} e^{-N p_{r}}(1+o(1)) .
$$

Утверждение теоремы 6 следует отсюда, из леммы 2 и соотношения (73).

Доказательство теоремы 7. Заметим, что

$$
k=N p_{r}+u_{r} \sigma_{r r} \sqrt{N}
$$

где $\left|u_{r}\right| \leqslant C<\infty$. Хорошо известно, что при $N p_{r}\left(1-p_{r}\right) \rightarrow \infty$ равномерно относительно таких значений $u_{r}$

$$
\left(\begin{array}{l}
N \\
k
\end{array}\right) p_{r}^{k}\left(1-p_{r}\right)^{N-k}=\frac{1+o(1)}{\sqrt{2 \pi N p_{r}\left(1-p_{r}\right)}} \exp \left\{-\frac{\left(k-N p_{r}\right)^{2}}{2 N p_{r}\left(1-p_{r}\right)}\right\}
$$

При доказательстве теорем 1, 2 и 3 показано, что в условиях теоремы 7 выполнено соотношение (63). Пусть $r \geqslant 2$. Справедливость соотношения (70) в случае $n / N \leqslant C<\infty$ следует из лемм 7,8 , а в случае $n / N \rightarrow \infty, N / \sqrt{n} \rightarrow \infty$ это соотношение можно установить, используя (3), (4), (6), (9), (15), (65), (71) и лемму 9. Для завершения доказательства теоремы 7 при $r \geqslant 2$ теперь достаточно применить лемму 2 , (9), (10), (63), (70), (71), (76).

Если $r=0$ или $r=1$, то доказательство проводится аналогично с учетом замечаний 1 и 2 к леммам 7 и $8,(61)$ и того, что в случае $r=0$ соотношение $\lambda(N-k) \rightarrow \infty$ верно, если $n^{2} / N \rightarrow \infty$, а в случае $r=1$ соотношение $\lambda^{3}(N-k) \rightarrow \infty$ выполняется при $n^{3} / N^{2} \rightarrow \infty$.

Доказательство теоремы 8. В случае $0<C_{1} \leqslant N / \sqrt{n} \leqslant C_{2}<\infty$ теорема 8 доказана в [7]. Пусть $N / \sqrt{n} \rightarrow 0$. Из (32) и (69) находим, что при фиксированных $t$

$$
\begin{aligned}
& \varphi^{N-k}\left(2 t /\left(b^{2} R N^{2}\right)\right) \rightarrow \exp \left\{-\left(1-p_{r}\right) \sqrt{-2 i t}\right\}, \\
&\left(1+\frac{p_{r}}{1-p_{r}}\left(1-\varphi^{-1}\left(\frac{2 t}{b^{2} R N^{2}}\right) \exp \left\{\frac{2 i t r}{b^{2} R N^{2}}\right\}\right)\right)^{N-k} \rightarrow \exp \left\{p_{r} \sqrt{-2 i t}\right\} .
\end{aligned}
$$

Пусть $\psi_{r}(t)$ - характеристическая функция случайной величины $2 \zeta_{N-k}^{(2)} /\left(b^{2} R N^{2}\right)$. Из (30) и (77) следует, как нетрудно видеть, что

$$
\psi_{r}(t) \rightarrow \exp \{-\sqrt{-2 i t}\}
$$

Последнее выражение является характеристической функцией устойчивого закона с показателем $\alpha=1 / 2$. Это значит, что для суммы $\zeta_{N-k}^{(2)}$ выполнены условия леммы 10 . Из этой леммы и (68) получаем соотношение (73), и нам осталось применить лемму 2 и (76). 
Доказательство теоремы 9. Если $N / \sqrt{n} \rightarrow \infty$, то, используя (2), (6), (8), (9), (69), (71), нетрудно убедиться, что выполнены условия лемм 7,8 , и с их помощью получить соотношения (63), (70), (72). Из этих соотношений следует, что при $r \rightarrow \infty$ выполняются (62), (73), и утверждение теоремы вытекает из леммы 2 и (74).

При $0<C_{1} \leqslant N / \sqrt{n} \leqslant C_{2}<\infty$ теорема 9 доказана в [7].

Пусть $N / \sqrt{n} \rightarrow 0$. Легко проверить, что соотношения (77), (78) справедливы и при $r \rightarrow \infty$, поэтому, как и при доказательстве теоремы 8, с помощью леммы 11 приходим к (73). Ясно, что $p_{r} \rightarrow 0$ при $r \rightarrow \infty$, поэтому из леммы 2 , (73) и (74) получаем утверждение теоремы 9.

\section{Список литературы}

1. Павлов Ю. Л., Предельные теоремы для числа деревьев заданного объема в случайном лесе. Матем. сб. (1977) 103, №3, 392-403.

2. Павлов Ю. Л., Асимптотическое распределение максимального объема дерева в случайном лесе. Теория вероятностей и ее применения (1977) 22, №3, 523-533.

3. Павлов Ю. Л., Один случай предельного распределения максимального объема дерева в случайном лесе. Матем. заметки (1979) 25, №5, 751-760.

4. Оттер Р. Число деревьев. В кн.: Перечислительные задачи комбинаторного анализа. Мир, Москва, 1979, с. 139-159.

5. Колчин В. Ф., Случайные графы. Физматлит, Москва, 2004.

6. Колчин В. Ф., О существовании гигантской компоненты в схемах размещения частиц. Обозрение прикладной и промышленной матем. (2000) 7, №1, 112-113.

7. Павлов Ю. Л., Предельные теоремы для объемов деревьев непомеченного графа случайного отображения. Дискретная математика (2004) 16, №3, 63-75.

8. Колчин А. В., Предельные теоремы для обобщенной схемы размещения. Дискретная математика (2003) 15, №4, 148-157.

9. Казимиров Н. И., О некоторых условиях отсутствия гигантской компоненты в обобщенной схеме размещения. Дискретная математика (2002) 14, №2, 107-118.

10. Казимиров Н. И., Об асимптотике больших компонент обобщенной схемы размещения. Труды Института прикладных матем. исследований КарНЦ РАН (2003) 4, 61-76.

11. Ткачук С. Г., Локальные предельные теоремы, учитывающие большие уклонения, в случае предельных устойчивых законов. Изв. АН Узб. ССР. Сер. физ.-матем. наук (1973) 2, 30-33.

Статья поступила 22.11.2004. 